\title{
Solution phase synthesis of halogenated graphene and the electrocatalytic activity for oxygen reduction reaction
}

\author{
Kuang-Hsu Wu, Da-Wei Wang a,b,*, Qingcong Zeng a , Yang Li a, Ian R. Gentle a,\# \\ a School of Chemistry and Molecular Biosciences, The University of Queensland, Brisbane 4072, Australia \\ ${ }^{\mathrm{b}}$ School of Chemical Engineering, UNSW Australia (The University of New South Wales), Sydney, NSW 2052, Australia
}

\section{A R T I C L E I N F O}

\section{Article history:}

Received 3 April 2014

Accepted 18 April 2014

Published 20 June 2014

\section{Keywords:}

Halogen-doping

Sulfur-doping

Graphene

Oxygen reduction reaction

Electrocatalyst

\begin{abstract}
A B S T R A C T
Metal-free carbon electrocatalyts for the oxygen reduction reaction (ORR) are attractive for their high activity and economic advantages. However, the origin of the activity has never been clearly elucidated in a systematic manner. Halogen group elements are good candidates for elucidating the effect, although it has been a difficult task due to safety issues. In this report, we demonstrate the synthesis of $\mathrm{Cl}-$, $\mathrm{Br}$ - and I-doped reduced graphene oxide through two solution phase syntheses. We have evaluated the effectiveness of doping and performed electrochemical measurements of the ORR activity on these halogenated graphene materials. Our results suggest that the high electronegativity of the dopant is not the key factor for high ORR activity; both $\mathrm{Br}$ - and I-doped graphene promoted ORR more efficiently than Cl-doped graphene. Furthermore, an unexpected sulfur-doping in acidic conditions suggests that a high level of sulfide can degrade the ORR activity of the graphene material.
\end{abstract}

(C) 2014, Dalian Institute of Chemical Physics, Chinese Academy of Sciences. Published by Elsevier B.V. All rights reserved.

\section{Introduction}

Since the invention of the hydrogen fuel cells in 1838, the electrocatalysis of the oxygen reduction reaction (ORR) has emerged as an attractive research topic, for it potentially allows enormous energy to be delivered for small mass [1]. The ORR reaction is critical for various types of fuel cells and metal-air batteries. Nowadays, much of the research focus is on developing a low cost and robust ORR electrocatalyst with Pt-grade activity while being environmentally benign [2,3]. Metal-free $\mathrm{N}$-doped carbon materials, in particular, have been discovered to offer the above advantages while delivering almost equivalent performance to Pt after thermal treatment [4]. However, the origin of electrocatalytic activity on N-doped carbons remains unclear due to a lack of understanding about the role that the dopant plays in the active site. This has prevented fur- ther development of metal-free ORR electrocatalyst and a solution should be actively pursued.

Recent research has established a hypothesis that the breaking of electroneutrality in the graphitic carbon lattice is responsible for forming active centers for ORR [5]. In order to validate this concept, an experiment would require a simple system which allows the dopant electronegativity to be tuned from the level of nitrogen down to a level close to carbon. To achieve this, halogen group elements are perhaps the ideal candidates since the electronegativity difference versus carbon $(\Delta \chi)$ for $\mathrm{Cl}, \mathrm{Br}$, and I are $0.61,0.41$, and 0.11 , respectively, by the Pauling scale. Unfortunately, current methods of halogen doping into carbon typically require harsh reaction conditions (e.g., high temperature treatment or laser ablation) and the direct use of highly toxic gases [6-9]. It would certainly be desirable to make a solution phase halogenation method for carbon ma-

\footnotetext{
* Corresponding author. Tel: +61-2-9385 6143; E-mail: da-wei.wang@unsw.edu.au

\# Corresponding author. Tel: +61-7-3365 4800; E-mail: i.gentle@uq.edu.au

DOI: 10.1016/S1872-2067(14)60108-X | http://www.sciencedirect.com/science/journal/18722067 | Chin. J. Catal., Vol. 35, No. 6, June 2014
} 
terials under ambient conditions available.

In this report, we demonstrate two solution phase preparations of $\mathrm{Cl}-$, Br-, and I-doped reduced graphene oxide (RGO) in different solvent systems and compare the effectiveness of the two doping procedures. Importantly, the electrocatalytic activity for ORR is carefully evaluated for each halogenated graphene material.

\section{Experimental}

\subsection{Materials}

RGO was prepared by a thermal exfoliation method on graphite oxide, which was synthesized according to a reported modified Hummer's method [10]. All other chemicals $\mathrm{KCl}$ ( $\geq 99 \%), \mathrm{KBr}(\geq 99 \%)$, and $\mathrm{KI}(\geq 99 \%), \mathrm{NaClO}$ (12.5\% in water), concentrated $\mathrm{H}_{2} \mathrm{SO}_{4}(\geq 98 \%)$ and $\mathrm{CCl}_{4}$ were purchased from Ajax Finechem Pty Ltd and Sigma-Aldrich, and used as received.

\subsection{Preparation of halogenated $R G O(X-R G O)$}

\subsubsection{Preparation $\mathrm{X}$-RGO in concentrated $\mathrm{H}_{2} \mathrm{SO}_{4}$}

Halogen-containing solutions were firstly prepared by adding $0.1 \mathrm{~mol} / \mathrm{L}$ equivalent amount of $\mathrm{NaClO}, \mathrm{KBr}$ and $\mathrm{KI}$ into 10 $\mathrm{mL}$ of $10 \mathrm{~mol} / \mathrm{L} \mathrm{H}_{2} \mathrm{SO}_{4}$ in a $20 \mathrm{~mL}$ glass bottle. Note that $\mathrm{NaClO}$ was used instead of $\mathrm{KCl}$ because the chloride anion is too stable to be oxidized by the concentrated acid. In a typical preparation of X-RGO, $2.0 \mathrm{mg}$ of RGO was introduced into the halogen-containing acid solution and dispersed in an ultrasonic bath for 30 min. With the bottle capped, the solution was stirred at ambient conditions overnight. The modified RGO was then filtered and washed with water and ethanol, and finally dried at $40{ }^{\circ} \mathrm{C}$ for collection.

\subsubsection{Preparation of X-RGO in halogen-dissolved $\mathrm{CCl}_{4}$}

The procedure for RGO modification in halogen-dissolved $\mathrm{CCl}_{4}$ was similar to that in $\mathrm{H}_{2} \mathrm{SO}_{4}$ except a halogen gas $\left(\mathrm{X}_{2}\right)$ ex-


of concentrated $\mathrm{H}_{2} \mathrm{SO}_{4}$ was placed in a capped glass bottle and $0.1 \mathrm{~mol} / \mathrm{L}$ equivalent of a halide salt was added into the two-phase mixture and shaken vigorously to dissolve the $\mathrm{X}_{2}$ gas into the organic phase. Since $\mathrm{HCl}$ does not dissolve in the organic phase, an equivalent amount of $\mathrm{NaClO}$ was used instead to generate $\mathrm{Cl}_{2}$ gas. The $\mathrm{X}_{2}$-dissolved $\mathrm{CCl}_{4}$ was then extracted and transferred to another bottle for RGO modification. The modification procedure was as described previously.

\subsection{Characterization}

The materials were characterized mainly by X-ray photoelectron spectroscopy (XPS) and Fourier-transform infrared (FT-IR) spectroscopy. XPS was performed with a Kratos XPS spectrometer using a monochromated $\mathrm{Al} \mathrm{K \alpha}$ source. The survey scan and high-resolution scan were measured at a pass energy of 160 and $20 \mathrm{eV} / \mathrm{s}$, respectively. All spectra were corrected against the $\mathrm{C} 1 \mathrm{~s}(\mathrm{C}-\mathrm{C})$ peak at $284.8 \mathrm{eV}$ as a reference and the full-width at half-maximum (FWHM) values were held constant for all peaks. Transmission electron microscopy (TEM) was carried out on a JEOL 1010 microscope and the micrographs were taken at an accelerating voltage of $100 \mathrm{kV}$. Ultraviolet-Visible (UV-Vis) spectroscopy was used to confirm the identity of a particular halogen dissolved in $\mathrm{CCl}_{4}$ solvent. This was performed on an Agilent Technologies Cary 60 UV-Vis spectrophotometer.

\subsection{Electrochemical measurements}

The electrochemical measurements of ORR activity were carried out using a CHI920D bipotentiostat instrument coupled with a RRDE-3A electrode rotor (ALS Co., Ltd.). In a typical cell, a rotating Pt ring/glassy carbon (GC) disk electrode, a Pt wire counter electrode and a $\mathrm{HgO} / \mathrm{Hg}$ reference electrode stabilized in $0.1 \mathrm{~mol} / \mathrm{L} \mathrm{KOH}$ were employed in a three-electrode system. All electrochemical reactions were performed in an $\mathrm{O}_{2}$-saturated $0.1 \mathrm{~mol} / \mathrm{L} \mathrm{KOH}$ electrolyte; the $\mathrm{O}_{2}$ saturation was done by purging with the gas for at least $30 \mathrm{~min}$ before the measurements. Linear sweep voltammetry on a rotating ring-disk electrode (LSV-RRDE) was performed using the above setup at a sweep rate of $10 \mathrm{mV} / \mathrm{s}$, the electrode was rotated constantly at $1500 \mathrm{r} / \mathrm{min}$ and the ring potential was held at $+0.5 \mathrm{~V}$ versus the reference electrode. All the potentials were quoted with respect to the reference electrode. The collecting efficiency of the RRDE was determined by using a bare glassy carbon disk in an Ar-saturated standard ferricyanide electrolyte $(5 \mathrm{mmol} / \mathrm{L}$ potassium ferricyanide in $0.1 \mathrm{~mol} / \mathrm{L} \mathrm{KNO}_{3}$ solution) under a rotation rate of $1500 \mathrm{r} / \mathrm{min}$. The collecting efficiency was found to be $\sim 0.32$ by calculating the ratio of the ring current (at oxidizing potential) and the disk current (at reducing potential) for the one-electron transfer reaction.

\section{Results and discussion}

In the preparation of X-RGO, concentrated $\mathrm{H}_{2} \mathrm{SO}_{4}$ was employed to generate $\mathrm{X}_{2}$ gases at laboratory scale from the various halide sources. Each reaction follows one of the chemical equations shown below:

$$
\begin{aligned}
& 2 \mathrm{NaClO}_{2} \mathrm{H}_{2} \mathrm{SO}_{4} \rightarrow 2 \mathrm{NaSO}_{4}+\mathrm{Cl}_{2(\mathrm{~g})}+2 \mathrm{H}_{2} \mathrm{O} \\
& \mathrm{H}_{2} \mathrm{SO}_{4}+2 \mathrm{H}^{+}+2 \mathrm{Br}^{-} \rightarrow \mathrm{Br}_{2(\mathrm{~g})}+\mathrm{SO}_{2(\mathrm{~g})}+2 \mathrm{H}_{2} \mathrm{O} \\
& \mathrm{H}_{2} \mathrm{SO}_{4}+8 \mathrm{H}^{+}+8 \mathrm{I}^{-} \rightarrow 4 \mathrm{I}_{2(\mathrm{~g})}+\mathrm{H}_{2} \mathrm{~S}_{(\mathrm{g})}+4 \mathrm{H}_{2} \mathrm{O}
\end{aligned}
$$

In this way, the highly oxidative $\mathrm{X}_{2}$ gases can react with $\mathrm{RGO}$ in the solution phase and introduce the desired $\mathrm{C}-\mathrm{X}$ species. Since halogens in their gaseous form are typically unstable in an aqueous medium, we have examined the effectiveness of this chemical modification in both $\mathrm{H}_{2} \mathrm{SO}_{4}$ and $\mathrm{CCl}_{4}$. Figure 1 (a) is the schematic diagram of the X-RGO preparation procedures in the two media. The typical sheet-like morphology of the raw RGO material was confirmed by TEM and is shown in Fig. 1(b). The presence of $\mathrm{X}_{2}$ gases in $\mathrm{CCl}_{4}$ after the solvent extraction was also identified by their characteristic absorption bands in the UV-Vis spectra as shown in Fig. 1(c); the excitation wavelengths for $\mathrm{Cl}_{2}, \mathrm{Br}_{2}$, and $\mathrm{I}_{2}$ are respectively 346, 414, and 512 $\mathrm{nm}$, which are in agreement with the reported values [11,12].

Composition analysis of the surface of the modified X-RGO requires a highly sensitive analytical technique and XPS was 
(a)
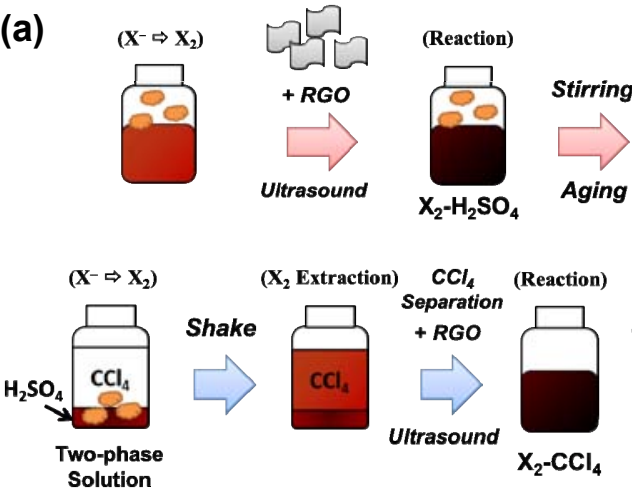
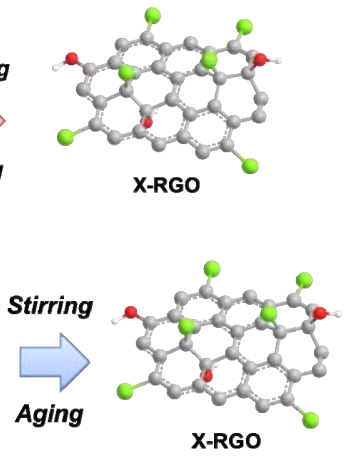
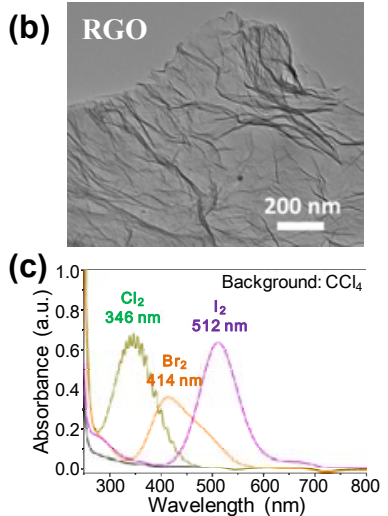

Fig. 1. (a) A schematic diagram of the preparation procedures of $\mathrm{X}-\mathrm{RGO}$ in $\mathrm{H}_{2} \mathrm{SO}_{4}$ (top) and $\mathrm{CCl}_{4}$ (bottom). (b) A typical TEM image of the $\mathrm{RGO}$ used in the synthesis. (c) UV-Vis spectra of halogen gases dissolved in $\mathrm{CCl}_{4}$.

hence selected for this purpose. Figure 2 displays the survey spectra of the X-RGO prepared in both aqueous acidic and organic media; all spectral intensity was normalized according to the $\mathrm{C} 1 \mathrm{~s}$ peaks. At the first glance, there is a fair amount of oxygen (8.0 at\%-9.0 at\%) observed in the raw RGO and this is found to be unchanged after the halogenation. No other impurities except for sulfur and the halogens could be identified in the spectra. In $10 \mathrm{~mol} / \mathrm{L}$ sulfuric acid (Fig. 2(a)), the product X-RGO shows almost negligible halogen content after the reaction. Interestingly, a notable amount of sulfur was unexpectedly incorporated into the I-RGO; this is probably due to the presence of $\mathrm{H}_{2} \mathrm{~S}$ after the iodide reduction of $\mathrm{H}_{2} \mathrm{SO}_{4}$ (Eq. (3)). In contrast, the reactions performed in $\mathrm{CCl}_{4}$ demonstrated a
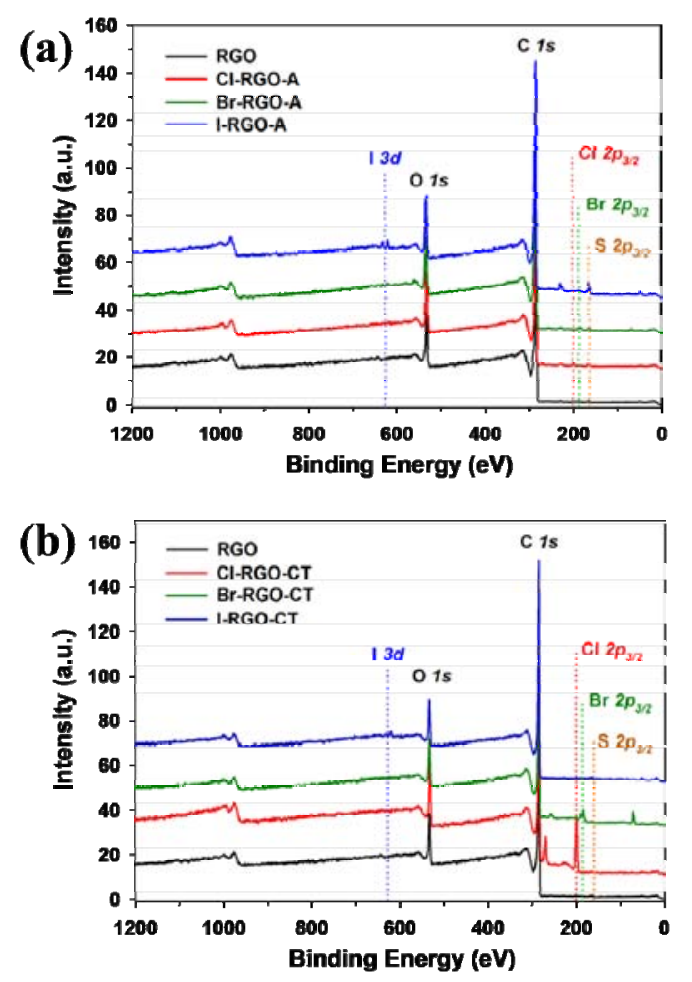

Fig. 2. XPS survey spectra for X-RGO prepared in $10 \mathrm{~mol} / \mathrm{L}$ sulfuric acid (a) and $\mathrm{CCl}_{4}$ media (b); the quantitative analysis of each element is given in Table 1. much-improved effect of raising the halogen content in the X-RGO. In Fig. 2(b), the $\mathrm{Cl} 2 p$ and the $\mathrm{Br} 2 p$ features display pronounced signals for the $\mathrm{Cl}-\mathrm{RGO}$ and the $\mathrm{Br}-\mathrm{RGO}$, respectively, although the iodine signature in the I-RGO remains at a very weak intensity. The quantitative results are tabulated in Table 1. For clarity, the X-RGO samples prepared in $10 \mathrm{~mol} / \mathrm{L} \mathrm{H}_{2} \mathrm{SO}_{4}$ are denoted $\mathrm{X}-\mathrm{RGO}-\mathrm{A}$ and those prepared in $\mathrm{CCl}_{4}$ are labelled $\mathrm{X}$-RGO-CT.

The quantitative analysis shows that the $\mathrm{S}$ content in the original RGO is only around 0.40 at\%, which arises from both sulfide and sulfate groups after the thermal exfoliation process (not shown). This low amount of S is likely to come from the Hummer's method of graphite oxide synthesis and it is therefore not surprising to find sulfur traces in the RGO. For the same reason, the reactions performed in $\mathrm{H}_{2} \mathrm{SO}_{4}$ show slightly increased $\mathrm{S}$ contents in the modified X-RGO; the special case of I-RGO-A will be discussed later. Furthermore, the remarkably high amount of incorporated $\mathrm{Cl}$ at 8.10 at\% in Cl-RGO-CT has shown that the reaction system is very effective when performed in an organic solvent, even under ambient conditions. However, it was not clear about the origin of the marginally increased oxygen level, given the fact that the $\mathrm{NaClO}$ salt was insoluble in $\mathrm{CCl}_{4}$. Similarly, Br-RGO-CT also yielded a fair amount of $\mathrm{Br}$ in the product, but the results were less encouraging for I-RGO-CT. This is likely because $\mathrm{I}_{2}$ gas is quite unreactive and the iodine groups are excellent leaving groups compared to other halogen elements. By all accounts, $\mathrm{H}_{2} \mathrm{SO}_{4}$ does not seem to be a suitable medium for the halogen modification of RGO, although the side reaction with iodide ion has effectively facilitated the sulfur doping of the graphene material.

Table 1

Composition analysis of the X-RGO prepared from the two methods.

\begin{tabular}{lcccc}
\hline \multirow{2}{*}{ Sample } & \multicolumn{4}{c}{ Composition (at\%), $\pm 0.2 \%$} \\
\cline { 2 - 5 } & $\mathrm{C}$ & $\mathrm{X}$ & $\mathrm{O}$ & $\mathrm{S}$ \\
\hline RGO & 91.58 & 0.0 & 8.02 & 0.40 \\
Cl-RGO-A & 90.19 & $0.31(\mathrm{Cl})$ & 8.78 & 0.72 \\
Br-RGO-A & 90.46 & $0.28(\mathrm{Br})$ & 8.60 & 0.66 \\
I-RGO-A & 88.72 & $0.14(\mathrm{I})$ & 8.40 & 2.74 \\
Cl-RGO-CT & 82.00 & $8.10(\mathrm{Cl})$ & 9.60 & 0.30 \\
Br-RGO-CT & 89.86 & $1.70(\mathrm{Br})$ & 8.07 & 0.37 \\
I-RGO-CT & 91.68 & $0.13(\mathrm{I})$ & 7.71 & 0.48 \\
\hline
\end{tabular}


Of course it is not assured that all incorporated halogen species are in the desired C-X configuration. We thus employed high-resolution XPS spectroscopy to examine the particular elements of interest. Figure 3 shows the high-resolution XPS spectra of the halogen and the sulfur peaks in X-RGO prepared in the acid system. Although the samples after modification were extensively rinsed with water and ethanol to remove possible impurities, the deconvoluted spectra show that a low to moderate level of halide species was present together with the covalently bonded halogen groups. In the $\mathrm{Cl} 2 p$ spectrum for Cl-RGO-A (Fig. 3(a)), the spin-orbit splitting between $2 p_{3 / 2}$ and $2 p_{1 / 2}$ was found to be $1.6 \mathrm{eV}$. The $2 p_{3 / 2}$ peaks of the two $\mathrm{Cl}$ species are located at 200.5 and $197.9 \mathrm{eV}$, corresponding to $\mathrm{C}-\mathrm{Cl}$ and $\mathrm{NaCl}$, respectively $[6,13]$. The halide may be sourced from the $\mathrm{Cl}_{2}$ decomposition in water and the reaction by-products. Clearly, the majority of the doped chlorine is in the form of C-Cl groups. The Br $3 d$ spectrum for Br-RGO-A (Fig. 3(b)) also has two $\mathrm{Br}$ species at 70.6 and $67.9 \mathrm{eV}$ of the $3 d_{5 / 2}$ feature, corresponding to $\mathrm{C}-\mathrm{Br}$ and $\mathrm{KCl}$, respectively $[14,15]$. The spin-orbit splitting for $\mathrm{Br} 3 d$ is about $1.4 \mathrm{eV}$. In this sample, the amount of C-Br was only about $66.5 \%$ of the total Br content. For I-RGO-A, the two peaks in I $3 d$ spectrum are associated with $3 d_{5 / 2}$ and $3 d_{3 / 2}$, with a spin-orbital splitting value of $11.6 \mathrm{eV}$ (Fig. 3(c)). Again, the C-I and KI species can respectively be found at 620.2 and $618.6 \mathrm{eV}[16,17]$, and only $41.2 \%$ out of the total I content is the $\mathrm{C}-\mathrm{I}$ group.

In an attempt to understand the reason behind the unexpected increase of sulfur content in I-RGO-A, the high-resolution $\mathrm{S} 2 p$ spectra of all X-RGO prepared in the acid system were carefully compared. In the spectra of X-RGO-A, two dominant
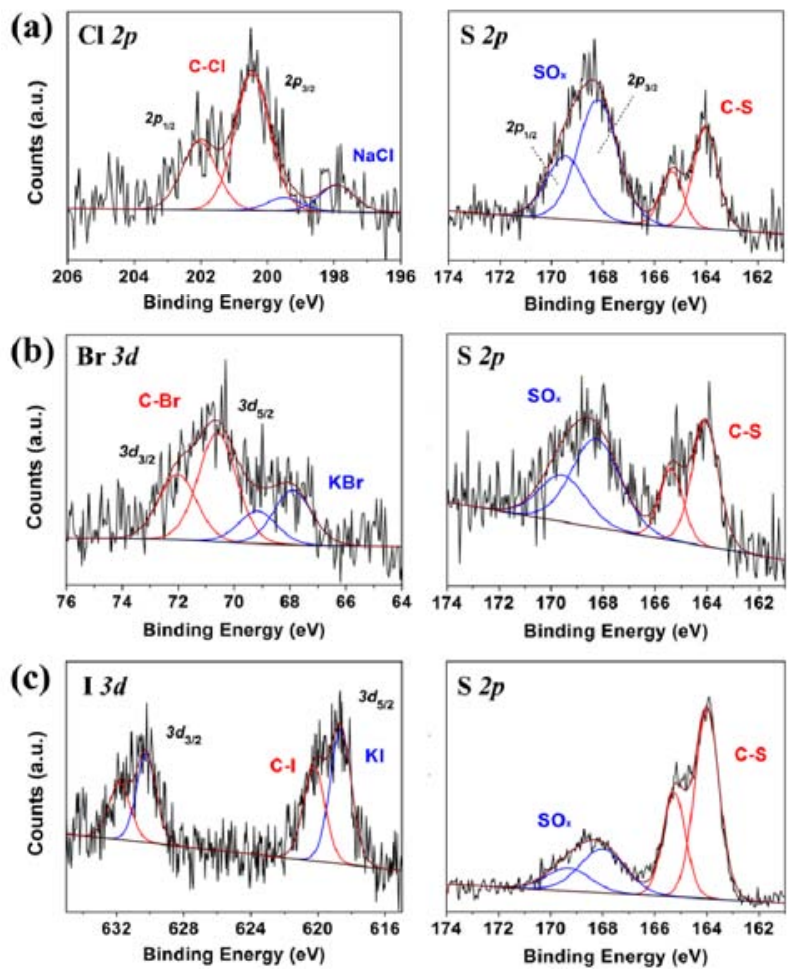

Fig. 3. XPS high-resolution spectra of the halogen and sulfur species for $\mathrm{Cl}-\mathrm{RGO}(\mathrm{a}), \mathrm{Br}-\mathrm{RGO}$ (b), and I-RGO (c) prepared in $10 \mathrm{~mol} / \mathrm{L} \mathrm{H}_{2} \mathrm{SO}_{4}$. types of sulfur can be identified, which are sulfide (C-S, 164.0 $\mathrm{eV})$ and sulfate/sulfonate groups ( $\left.\mathrm{SO}_{x}, 168.2 \mathrm{eV}\right)$ at the $2 p_{3 / 2}$ positions $[18,19]$. In the case of Cl-RGO-A and Br-RGO-A, the $\mathrm{C}-\mathrm{S}$ and $\mathrm{SO}_{x}$ level were roughly in equal amounts. As aforementioned, these sulfides should come from the raw RGO material. In contrast, the $\mathrm{S}$ in I-RGO-A is predominantly in the form of sulfide as shown in the $\mathrm{S} 2 p$ spectrum (Fig. 3(c)). Since there is no direct sulfide source in the starting reagents, the excessive sulfide must be the consequence of additional reactions occurring during the RGO modification process, likely by the $\mathrm{H}_{2} \mathrm{~S}$ from the sulfate reduction. This result has confirmed that I-RGO-A is really a sulfide-doped material and $\mathrm{H}_{2} \mathrm{~S}$ is perhaps an excellent reactant for sulfur doping.

The high-resolution XPS spectra of the halogens in X-RGO-CT are presented in Fig. 4. As the reactions were carried out in a non-polar electrolyte, the problem of salt deposition on the materials was entirely avoided. In the $\mathrm{Cl} 2 p$ spectrum of Cl-RGO-CT (Fig. 4(a)), the neat spectrum with a $2 p_{3 / 2}$ peak positioned at $200.3 \mathrm{eV}$ directly indicates the introduction of $\mathrm{C}-\mathrm{Cl}$ into $\mathrm{RGO}$ by $\mathrm{Cl}_{2}$. The $\mathrm{Br} 3 d$ spectrum for $\mathrm{Br}-\mathrm{RGO}-\mathrm{CT}$ reveals one bromine type at $70.4 \mathrm{eV}$, suggesting the presence of $\mathrm{C}-\mathrm{Br}$ group (Fig. 4(b)). The I $3 d$ spectrum for I-RGO-CT is shown in Fig. 4(c). The position of the $3 d_{5 / 2}$ peak is at $620.0 \mathrm{eV}$, signifying the presence of C-I group despite of the broader peak width. This may be due to the minor contamination by $\mathrm{I}_{2}$ given that the binding energy for C-I ( $620.2 \mathrm{eV})$ and $\mathrm{I}_{2}(619.9 \mathrm{eV})$ is too close to be distinguished $[16,20]$. Nonetheless, all of the X-RGO-CT samples have definite $\mathrm{C}-\mathrm{X}$ signatures and are free from halide
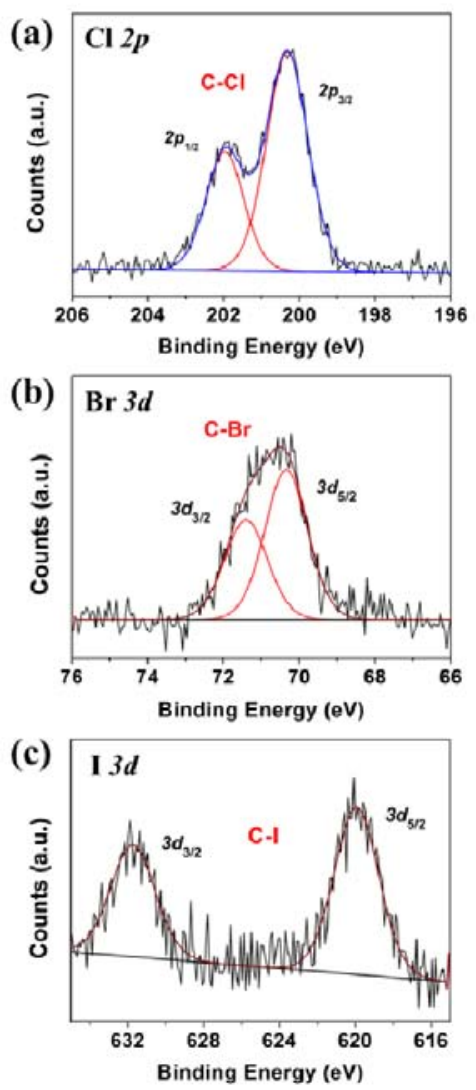

Fig. 4. XPS high-resolution spectra of the halogen species for Cl-RGO (a), Br-RGO (b), and I-RGO (c) prepared in $\mathrm{CCl}_{4}$. 
contamination.

With the above data, we are able to summarize the $\mathrm{C}-\mathrm{X}$ and $\mathrm{C}-\mathrm{S}$ contents in the modified RGO, as shown in Fig. 5. Briefly, the $\mathrm{C}-\mathrm{Cl}$ content in $\mathrm{Cl}-\mathrm{RGO}$ and the $\mathrm{C}-\mathrm{Br}$ content in Br-RGO are probably the most pronounced differences for the acid and organic systems. The minor amount of the water-soluble halide salts that present in a non-covalently bonded fashion should have no influence on the electrocatalytic activity in an aqueous electrolyte system. I-RGO-CT can be regarded as a true iodine-modified RGO, whereas the considerable number of C-S groups in I-RGO-A means that it is in fact better described as a sulfur-modified RGO. By using electrochemical methods, we shall be able to clarify the influence of each dopant on oxygen reduction electrocatalysis.

To study the electrocatalytic activity of the modified graphene materials, the ORR was carried out in an oxygen-saturated $0.1 \mathrm{~mol} / \mathrm{L} \mathrm{KOH}$ electrolyte. In general, ORR propagates through a two-step reaction in which each individual step is a two-electron reduction process [21]. The following chemical equations describe the elementary steps of ORR:

$$
\begin{aligned}
& \mathrm{O}_{2}+\mathrm{H}_{2} \mathrm{O}+2 \mathrm{e}^{-} \rightarrow \mathrm{HO}_{2}^{-}+\mathrm{OH}^{-} \\
& \mathrm{HO}_{2}+\mathrm{H}_{2} \mathrm{O}+2 \mathrm{e}^{-} \rightarrow 3 \mathrm{OH}^{-}
\end{aligned}
$$

An efficient ORR process would initiate the reaction at low reaction overpotential (i.e., towards positive onset potential) and deliver a large reaction current [3]. In an ideal scenario, an apparent four-electron ORR can be attained when the secondary reaction is sufficiently fast that the escape of the peroxide intermediate from the electrode surface can be prevented [21]. By incorporating halogen group elements into graphene, we are able to investigate the effect of increasing dopant electronegativity on ORR electrocatalytic activity using a metal-free carbon platform.

We have employed a linear sweep voltammetry technique using a rotating ring-disk electrode (LSV-RRDE) to study the electrocatalytic behavior of X-RGO. This technique basically creates a hydrodynamic environment which promotes the introduction of fresh reactant and the removal of reaction products from the electrode surface. Any meta-stable intermediate such as peroxide anion, if generated, will be detected at the ring electrode generating a ring current. Figure 6 is the LSV-RRDE profiles of X-RGO from the two preparation conditions; the unmodified RGO was used as a reference for comparison. In the

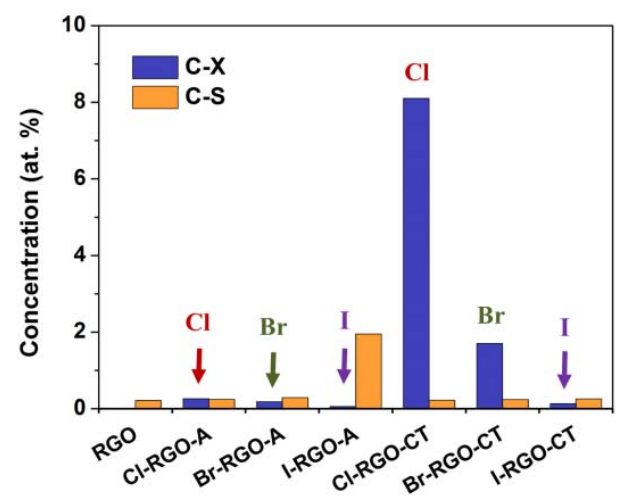

Fig. 5. The $\mathrm{C}-\mathrm{X}$ and $\mathrm{C}-\mathrm{S}$ content in the $\mathrm{X}-\mathrm{RGO}$ from two solution methods derived semi-quantitatively from XPS.
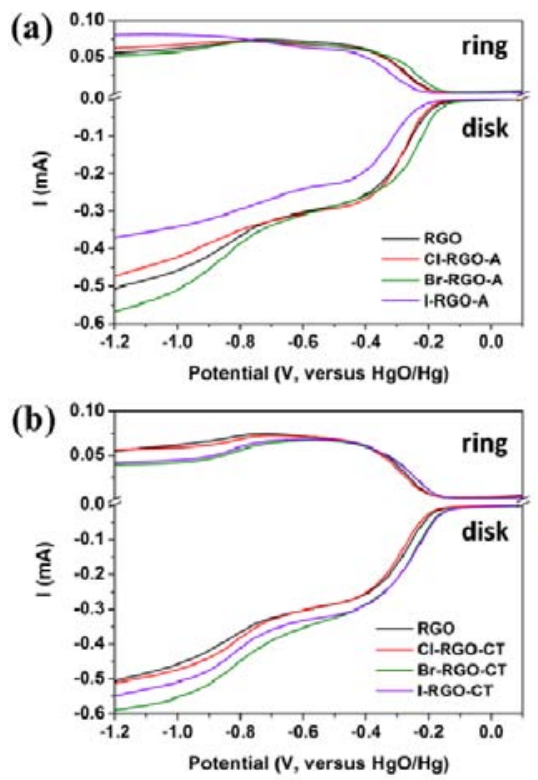

Fig. 6. LSV-RRDE profiles of the X-RGO in acid system (a) and in $\mathrm{CCl}_{4}$ system (b) in $\mathrm{O}_{2}$-saturated $0.1 \mathrm{~mol} / \mathrm{L} \mathrm{KOH}$ electrolyte.

voltammogram of the raw RGO, a two-step profile starting from $-0.15 \mathrm{~V}$ can be read from the disk current, suggesting the occurrence of the two-step consecutive reaction mechanism. The consistent initial increase and the decrease of the ring current at approximately $-0.80 \mathrm{~V}$ support the view that the reaction mechanism is in agreement with equation (1) and (2). On this basis, it is clear that the Cl-RGO-A in Fig. 6(a) shows a similar reaction behavior while starting at an almost identical onset potential to RGO. This means the Cl-doping does not affect RGO in a way that alters the ORR property of the electrocatalyst. Similar reaction features can be observed for Cl-RGO-CT (Fig. 6(b)). This affirms that the doped $\mathrm{Cl}$ does not participate in the ORR, even at a much higher concentration. Br-doping, on the other hand, displays less negative reaction onset potential at $-0.12 \mathrm{~V}$, as can be read from Br-RGO-A and Br-RGO-CT. This suggests the doped $\mathrm{Br}$ promotes the $\mathrm{O}_{2}$ reduction into $\mathrm{HO}_{2}{ }^{-}$at a lower energy cost. In addition, Br-RGO-CT shows an improvement in the reaction current as compared to Br-RGO-A; this could simply be a direct result of increased $\mathrm{Br}$ content. Looking at I-RGO-CT as a real I-doped RGO as shown in Fig. 6(b), the improved reaction behavior in the onset potential and the reaction current has also suggested the I-doping of RGO is beneficial for ORR, despite the low iodine concentration in the sample. Yet, it is not clear why Br-doped and I-doped RGO share a similarly improved reaction behavior when there is a difference in the dopant electronegativity. Nevertheless, both $\mathrm{Br}-$ and I-doped RGO demonstrate a better ORR activity in terms of the onset potentials and the reaction currents over the entire reaction potential range, as compared to Cl-doped RGO. Therefore, it is suggested that the difference in electronegativity between the dopant and the neighboring carbon atom is not the crucial factor in providing excellent activity for ORR.

As a practically S-doped graphene material, I-RGO-A in Fig. 6(a) exhibits a poor reaction current with a strongly negative 
onset potential of around $-0.20 \mathrm{~V}$. Moreover, the ring current shows a steady increase instead of a reduction at low potential. This suggests that the reaction is producing multiple intermediate species which can be easily oxidized at the Pt ring. This will reduce the electron gain per reaction and lead to poor energy efficiency in a fuel cell. Clearly the incorporated sulfide groups in I-RGO-A have an adverse effect on the general ORR activity of the electrode. Although there are reports about the beneficial effects of doping sulfur by a high temperature treatment [22], our results of doping sulfur at ambient temperature do not support this conclusion.

In an evaluation of ORR performance for each doped RGO material, we have calculated the electron transfer $(n)$ number based on the following equation [23], where $I_{\mathrm{d}}$ is the disk current, $I_{\mathrm{r}}$ is the ring current and $N$ denotes the collecting efficiency at the ring electrode.

$$
n=\frac{4 I_{\mathrm{d}}}{I_{\mathrm{d}}+\frac{I_{\mathrm{r}}}{N}}
$$

As shown in Fig. 7, all modified RGO materials except for I-RGO-A have $n$ numbers of less than 2.5 at low negative potential that increase at approximately $-0.80 \mathrm{~V}$. The sulfur-doped RGO (I-RGO-A) performs ORR at an electron transfer number of around 2.0, suggesting that the secondary reduction of the peroxide intermediate is inhibited. In other words, the sulfide groups at the electrode surface likely repel the intermediate species as soon as they are formed. The Cl-RGO samples produced by both doping methods share similar $n$ number profiles across the potential range and both are similar to the reference RG0. The $n$ numbers are around $\sim 2.8$ at $-1.0 \mathrm{~V}$, which indicates that only a small portion of peroxide intermediate has been consumed for a full reduction of $\mathrm{O}_{2}$. Next, both $\mathrm{Br}-\mathrm{RGO}$ show a clear improvement in $n$ number over the reaction potential range. This means a higher percentage of the intermediate is utilized, especially for Br-RGO-CT; an $n$ number of $\sim 3.2$ can be reached at $-1.0 \mathrm{~V}$. A similar $n$ number profile is also found for I-RGO-CT, which may suggest that the ORR activity by the electron transfer efficiency is at a comparable level with Br-RGO-CT. We are unable to distinguish between Br-doped and I-doped RGO in terms of which really has a superior activity from the limited sample size.

Overall our results have established an understanding of the

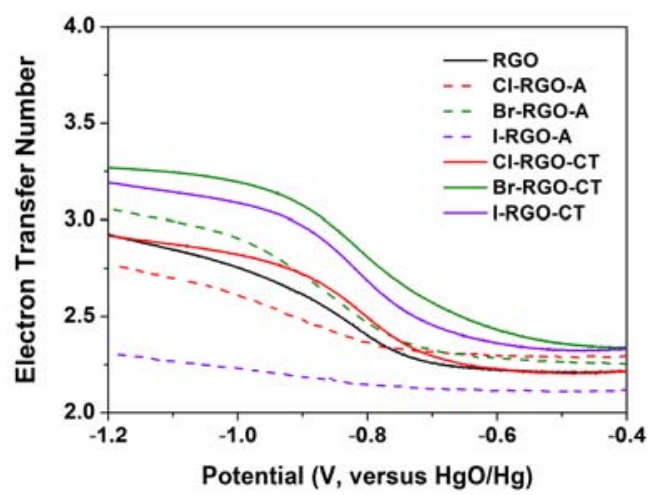

Fig. 7. The electron transfer number evolution on X-RGO across the ORR potentials range. impact of doping highly electronegative elements into carbon on ORR performance. The ORR activity in the view of general performance (i.e., onset potential, reaction current and $n$ number) of the heteroatom modified RGO can be ranked approximately as: $\mathrm{Br}-\mathrm{RGO} \approx \mathrm{I}-\mathrm{RGO}>\mathrm{Cl}-\mathrm{RGO} \approx \mathrm{RGO}>\mathrm{S}$-RGO. Clearly, a highly electronegative dopant does not necessarily create ORR active sites as in the case of N-doped carbons. Firstly, none of the doped halogen elements led to an apparent four-electron ORR. Secondly, $\mathrm{Cl}$ exhibits the highest electronegativity among the halogen group elements studied here, but it does not provide an enhanced electrocatalytic activity. Hence, the origin of ORR activity is perhaps rather related to the local electronic and bonding configuration around the dopant in the carbon lattice [24]. Yet, the dopant electronegativity may still have a role in tuning the electronic configuration. For instance, our Br-RGO has yielded improved activity, noting that the electronegativity value of bromine is very close to that of nitrogen $\left(\chi_{\mathrm{Br}}\right.$ $=2.96$ and $\chi_{\mathrm{N}}=3.04$ ). How such a level of electronegativity affects the electronic configuration will require further research. Overall, we believe that this report has solved an important piece of the puzzle about the origin of ORR activity on metal-free carbon electrocatalysts.

\section{Conclusions}

We have successfully demonstrated the preparation of halogenated graphene in both $\mathrm{H}_{2} \mathrm{SO}_{4}$ and $\mathrm{CCl}_{4}$ media and addressed the effects of doping RGO with $\mathrm{Cl}, \mathrm{Br}$, I, and S elements on the ORR activity. In $10 \mathrm{~mol} / \mathrm{L} \mathrm{H}_{2} \mathrm{SO}_{4}$, the reaction yield of $\mathrm{C}-\mathrm{X}$ groups was very poor (less than 0.5 at $\%$ ) and a considerable amount of sulfide was incorporated into I-RGO-A by a side reaction involving $\mathrm{H}_{2} \mathrm{~S}$. For reactions performed in non-polar $\mathrm{CCl}_{4}$ medium, the halogen introduction was highly effective and free of possible halide impurities; the $\mathrm{Cl}$ content in Cl-RGO-CT was as high as 8.1 at $\%$. The electrochemical experiments revealed that both Br-RGO and I-RGO facilitated a more efficient ORR than Cl-RGO and the undoped RGO at a higher reaction onset potential. The sulfide-containing I-RGO-A delivered the worst general ORR activity among all the modified RGO materials. The level of activity can be approximately rated in the order: $\mathrm{Br}-\mathrm{RGO} \approx \mathrm{I}-\mathrm{RGO}>\mathrm{Cl}-\mathrm{RGO} \approx \mathrm{RGO}>\mathrm{S}$-RGO. It is clear that high electronegativity of a dopant is not the key factor for a superior ORR electrocatalyst.

\section{Acknowledgments}

We acknowledge financial support from The University of Queensland and the Australian Research Council Discovery Project (DP110100550). The authors acknowledge the facilities, and the scientific and technical assistance of the Australian Microscopy \& Microanalysis Research Facility at the Centre for Microscopy and Microanalysis, The University of Queensland.

\section{References}

[1] Hoogers G. Fuel Cell Technology Handbook. CRC Press, 2002. 1-1

[2] Wang B. J Power Sources, 2005, 152: 1 


\section{Graphical Abstract}

Chin. J. Catal., 2014, 35: 884-890 doi: 10.1016/S1872-2067(14)60108-X

Solution phase synthesis of halogenated graphene and the electrocatalytic activity for oxygen reduction reaction

Kuang-Hsu Wu, Da-Wei Wang*, Qingcong Zeng, Yang Li, Ian R. Gentle*

The University of Queensland, Australia;

UNSW Australia (The University of New South Wales), Australia

Halogen-doped graphene can be simply doped in a mild solution phase reaction. The electrocatalytic activity for oxygen reduction reaction on halogen-doped graphene does not show a direct dependence on the electronegativity of the halogen dopant.
ORR Activity:

$\mathrm{Br}-\mathrm{RGO} \approx \mathrm{I}-\mathrm{RGO}>\mathrm{Cl}-\mathrm{RGO} \approx \mathrm{RGO}$

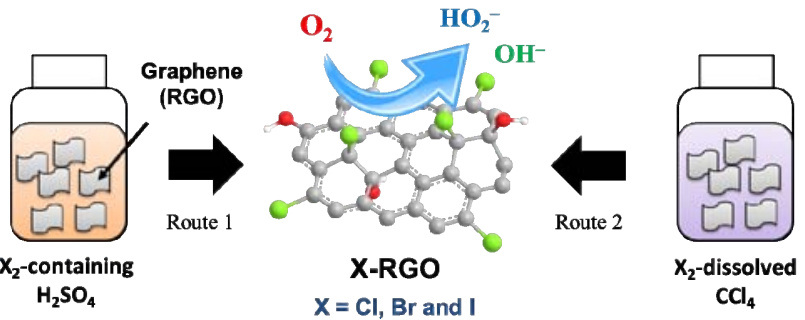

[3] Cheng F, Chen J. Chem Soc Rev, 2012, 41: 2172

[4] Gong K P, Du F, Xia Z H, Durstock M, Dai L M. Science, 2009, 323 : 760

[5] Zhou X, Tian Z, Li J, Ruan H, Ma Y, Yang Z, Qu Y. Nanoscale, 2014, 6: 2603

[6] Poh H L, Šimek P, Sofer Z, Pumera M. Chem Eur J, 2013, 19: 2655

[7] Zheng J, Liu H T, Wu B, Di C A, Guo Y L, Wu T, Yu G, Liu Y Q, Zhu D B. Sci Rep, 2012, 2: 662

[8] Wu J, Xie L, Li Y, Wang H, Ouyang Y, Guo J, Dai H. J Am Chem Soc, 2011, 133: 19668

[9] Lee W H, Suk J W, Chou H, Lee J, Hao Y, Wu Y, Piner R, Akinwande D, Kim K S, Ruoff R S. Nano Lett, 2012, 12: 2374

[10] Marcano D C, Kosynkin D V, Berlin J M, Sinitskii A, Sun Z, Slesarev A, Alemany L B, Lu W, Tour J M. ACS Nano, 2010, 4: 4806

[11] Seery D J, Britton D. J Phys Chem, 1964, 68: 2263

[12] Doskocil E J, Bordawekar S V, Kaye B G, Davis R J. J Phys Chem B, 1999, 103: 6277

[13] Park C D, Choi W Y, Kang H. Radiat Eff Defect S, 1990, 115: 65
[14] Zhou X L, Solymosi F, Blass P M, Cannon K C, White J M. Surf Sci, 1989, 219: 294

[15] Crist B V. Surf Sci Spectra, 1992, 1: 292

[16] Solymosi F, Révész K. Surf Sci, 1993, 280: 38

[17] Morgan W E, Van Wazer J R, Stec W J. J Am Chem Soc, 1973, 95 : 751

[18] Kelemen S R, George G N, Gorbaty M L. Fuel, 1990, 69: 939

[19] Yu X R, Liu F, Wang Z Y, Chen Y. J Electron Spectrosc Relat Phenom, 1990, 50: 159

[20] Dillard J G, Moers H, Klewe-Nebenius H, Kirch G, Pfennig G, Ache H J.J Phys Chem, 1984, 88: 4104

[21] Zhang J, Song C. Electrocatalytic Oxygen Reduction Reaction, PEM Fuel Cell Electrocatalysts and Catalyst Layers, Springer, 2008. 89

[22] Yang Z, Yao Z, Li G, Fang G, Nie H, Liu Z, Zhou X, Chen X, Huang S. ACS Nano, 2011, 6: 205

[23] Tan Y, Xu C, Chen G, Fang X, Zheng N, Xie Q. Adv Func Mater, 2012, 22: 4584

[24] Wang D W, Su D. Ener Environ Sci, 2014, 7: 576 